\title{
Drug repurposing of approved drugs Elbasvir, Ledipasvir, Paritaprevir, Velpatasvir, Antrafenine and Ergotamine for combating COVID19
}

\section{Vishal Mevada ${ }^{1}$, Pravin Dudhagara ${ }^{2}$, Himani Gandhi ${ }^{2}$, Nilam Vaghamshi ${ }^{2}$, Urvisha Beladiya $^{2}$ and Rajesh Patel $^{2}$}

\footnotetext{
${ }^{1}$ Department of Life Sciences, Hemchadracharya North Gujarat University, Patan, India

${ }^{2}$ Bioinformatics Laboratory \& Super Computing Facility, Department of Biosciences, Veer Narmad South Gujarat University, Surat, India
}

rkpatel@vnsgu.ac.in 


\begin{abstract}
Aims: Pneumonia of unknown cause detected in Wuhan, China was first reported to the WHO Country Office in China on 31 December 2019. The outbreak was declared a Public Health Emergency of International Concern on 30 January 2020. Currently, there is no Vaccine against COVID-19 pandemic and infection is spreading worldwide very rapidly. The present study aimed to meet the exigent requirement of practicable COVID19 drug treatment with a computational multitarget drug repurposing approach.
\end{abstract}

Main methods: Many reports are available with in silico drug repurposing. However, the majority of them engrossed on a single target. In the present study, 1735 FDA approved drugs screened with molecular docking approach against Covid19 protein and extracts the drug combination targeting COVID19 proteins comprehensively.

Key findings: The study designated Elbasvir, Ledipasvir, Paritaprevir, Velpatasvir, Antrafenine, and Ergotamine as promising drug candidates for covid19 treatment. The computational analysis also revealed the better potential of the proposed drug combination over the currently used drugs for COVID19 treatment.

Significance: The anticipated drug combination is acting on both non-structural and structural proteins therefore, it can be able to reduce the COVID19 infection process and also reduce viral multiplication. Moreover, the drugs are safe and well-known so it can be rapidly explored further for the COVID19 drug discovery process.

\title{
Keywords
}

Drug repurposing, Molecular Docking, COVID 19, Elbasvir, Ledipasvir, Paritaprevir, Velpatasvir, Antrafenine, Ergotamine.

\section{Introduction}

The World Health Organization announced in February 2020 that COVID-19 is the official name of the disease. World Health Organization chief Tedros Adhanom Ghebreyesus explained that CO stands for corona, VI for virus and D for disease, while 19 is for the year that the outbreak was first identified; 31 December 2019 [1]. Coronavirus disease 2019 (COVID-19) is a contagious disease caused by severe acute respiratory syndrome coronavirus 2 (SARS-CoV-2) previously referred to as the 2019 novel coronavirus (2019-nCoV) [2]. In 2019 at Wuhan, the capital of Hubei, China, the disease was first reported and then it spread worldwide, resulting in the 2019-20 coronavirus pandemic $[3,4]$. 
At present, there are no clinically proven vaccines and medicines for COVID-19 prevention and treatment as per U.S. Food and Drug Administration (FDA), the Centers for Disease Control and Prevention (CDC) and World Health Organization (WHO) [5]. On an interactive web-based dashboard to track COVID-19 in real-time as on April 19, 2020, in the entire world more than 200 countries/territories are having 2.3 Million confirmed cases and 1,61,283 deaths worldwide and more than 50000 increase daily reported since March 26, 2020 [6,7].

Recovery observed in patients of Covid19 treated with the mixture of Anti-HIV drugs like Libonavir \& Ritonavir, Anti-SARS drugs Oseltamivir and Anti-malarial drug Chloroquine in India (Wadhawan, 2020). In South Korea, human MERS-CoV successfully revokes the viral clearance using a combination of Lopinavir/Ritonavir (LPV/RTV) (Anti-HIV drugs) pegylated interferon and ribavirin [8]. Still, the treatment with anti-HIV drugs is a mystery for the Patients and Researchers as well [9]. As per data available on https://www.excelra.com/covid-19-drug-repurposing-database/data currently, 125 different drug molecules are reported under trial.

Recently, diverse computational approaches explored for screening the drug molecules for COVID19 treatment. Molecular docking of Lopinavir, Darunavir and Ritonavir reported against the modelled structure of COVID19 proteases, coronavirus endopeptidase C30 (CEP_C30) and papain-like viral protease (PLVP) [10]. MM-PBSA-WSAS (Molecular dynamics simulations followed by binding free energy calculations using an endpoint method) employed for Fast Identification of Possible Drug against COVID-19 protease [11]. Anti-HCV drugs, Sofosbuvir, IDX-184, Ribavirin, and Remidisvir reported promising drug candidates with a docking approach against modelled COVID-19 RNA dependent RNA polymerase (RdRp) [12]. Hirokawa et al., (2020) identified one hundred and several dozen potentially candidate drugs for $3 \mathrm{CL}$ protease inhibitors, which are already approved as antiviral, HIV protease inhibitors, antibacterial or antineoplastic agents with in silico docking-based screening approach, which combines molecular docking with a protein-ligand interaction fingerprint (PLIF) scoring method.

Many other reports are also available with in silico drug repurposing but the majority of them engrossed on a single target. The present study designed for docking FDA approved Drugbank compounds with molecular weight less than 700 against all COVID-19 experimentally and computationally generated protein structure.

\section{Materials and Methods}

\section{Target preparation}

Crystal structure of COVID-19 main protease in complex with an inhibitor N3 (PDB ID: 6LU7) [13], SARS-Coronavirus NSP12 bound to NSP7 and NSP8 co-factors(6NUR) [14] and Pre-fusion 2019- 
nCoV spike glycoprotein with a single receptor-binding domain (6VSB)[15] retrieved from the Protein Data Bank available at https://www.rcsb.org/. Additionally, computationally predicted 24 modelled structures based on protein sequences translated from the complete genome of Severe acute respiratory syndrome coronavirus 2 isolate Wuhan-Hu1 (Genbank Accession: MN908947.3) available on I-TASSAR online server also included in the present study (Table 1) [16]. Total 27 receptors threedimensional structures were subjected addition of Hydrogen for $\mathrm{pH} 7.0$ and Gasteiger charges were using Open Babel [17]. The resulting structures converted to PDBQT format using python script “prepare_receptor.py” from AutoDock Tools [18].

Table 1: Receptor molecules and their binding energy

\begin{tabular}{|c|c|c|c|c|c|}
\hline \multirow[b]{2}{*}{ No. } & \multirow[b]{2}{*}{ Source } & \multirow[b]{2}{*}{ Code } & \multirow[b]{2}{*}{ Description } & \multicolumn{2}{|c|}{ Binding Energy $(\mathrm{kcal} / \mathrm{mol})$} \\
\hline & & & & Overall range & $\begin{array}{l}\text { Range of top } 20 \\
\text { molecules }\end{array}$ \\
\hline 1 & Modeled & QHD43415_1 & Host translation inhibitor (nsp1) & -9.3 to -0.5 & -9.3 to -8 \\
\hline 2 & Modeled & QHD43415_2 & Non-structural protein 2 (nsp2) & -11.6 to -0.3 & -11.6 to -10.8 \\
\hline 3 & Modeled & QHD43415_3 & Papain-like proteinase & -12.4 to 1 & -12.4 to -11.1 \\
\hline 4 & Modeled & QHD43415_4 & Non-structural protein 4 (nsp4) & -13.4 to -0.1 & -13.4 to -10.9 \\
\hline 5 & Modeled & QHD43415_5 & Proteinase 3CL-PRO & -10.2 to -1 & -10.2 to -9 \\
\hline 6 & Modeled & QHD43415_6 & Non-structural protein 6 (nsp6) & -10 to -1 & -10 to -8.8 \\
\hline 7 & Modeled & QHD43415_7 & Non-structural protein 7 (nsp7) & -7.1 to 5 & -7.1 to -6.3 \\
\hline 8 & Modeled & QHD43415_8 & Non-structural protein 8 (nsp8) & -8.3 to 5 & -8.3 to -7 \\
\hline 9 & Modeled & QHD43415_9 & Non-structural protein 9 (nsp9) & -9.1 to -1 & -9.1 to -7.7 \\
\hline 10 & Modeled & QHD43415_10 & Non-structural protein 10 (nsp10) & -9.8 to -1 & -9.8 to -8.8 \\
\hline 11 & Modeled & QHD43415_11 & $\begin{array}{l}\text { RNA-directed RNA polymerase } \\
\text { (RdRp) }\end{array}$ & -11.5 to -1 & -11.5 to 9.8 \\
\hline 12 & Modeled & QHD43415_12 & Helicase (Hel) & -12.4 to -1.2 & -12.4 to -9.8 \\
\hline 13 & Modeled & QHD43415_13 & $\begin{array}{l}\text { Guanine-N7 methyltransferase } \\
(\text { ExoN) }\end{array}$ & -12 to -1.1 & -12 to -10.9 \\
\hline 14 & Modeled & QHD43415_14 & Endoribonuclease (NendoU) & -10.9 to -0.8 & -10.9 to -9.8 \\
\hline 15 & Modeled & QHD43415_15 & 2'-O-methyltransferase (2'-O-MT) & -10.9 to -1.2 & -10.9 to -9.8 \\
\hline 16 & Modeled & QHD43416 & Surface glycoprotein (S) & -10.8 to -0.6 & -10.8 to -9.8 \\
\hline 17 & Modeled & QHD43417 & Potassium sensitive ion channels & -9.8 to -1 & -9.8 to -8.9 \\
\hline 18 & Modeled & QHD43418 & E-Component (Self Assembly) & -8.2 to -0.8 & -8.2 to -7.4 \\
\hline 19 & Modeled & QHD43419 & M-Component (Morphogenesis) & -10 to -1.1 & -10 to -8.9 \\
\hline 20 & Modeled & QHD43420 & ORF6(Virulence) & -7.8 to -0.8 & -7.8 to -7 \\
\hline 21 & Modeled & QHD43421 & ORF7a (Tethering Suppression) & -8.5 to -1.1 & -8.5 to -7.6 \\
\hline 22 & Modeled & QHD43422 & ORF8 (Hypothetical) & -9.4 to -0.7 & -9.4 to -7.9 \\
\hline 23 & Modeled & QHD43423 & N-Component & -12.1 to -1.2 & -12.1 to -10.6 \\
\hline 24 & Modeled & QHI42199 & ORF10 & -4.5 to 5 & -4.5 to -3.8 \\
\hline 25 & PDB & 6LU7 & COVID-19 main protease & -9.7 to -0.9 & -9.7 to -9.1 \\
\hline 26 & PDB & 6 NUR & SARS-Coronavirus NSP12 & -10.8 to -0.7 & -10.8 to -9.7 \\
\hline 27 & PDB & $6 \mathrm{VSB}$ & 2019-nCoV spike glycoprotein & -9.5 to -0.6 & -9.5 to -8.3 \\
\hline
\end{tabular}




\section{Ligand preparation}

Total of 1735 approved drug molecules having a molecular weight of less than $700 \mathrm{MW}$ downloaded from Drugbank [19]. The drug structures downloaded in 2D-SDF format. The drug molecules subjected to optimization of Gasteiger charges and MMFF94 force-field using steepest descents followed by conjugate gradient minimization at 400 modes using OpenBabel and customized python script “prepare_ligand.py” script provided by AutoDockTool 4.0 utilities. Subsequently, the structures were converted to 3D-PDBQT format using MGL Tools for Autodock provided by The Scripps Research Institute.

\section{Virtual Screening}

Molecular docking of all 27 protein receptors against the approved 1735 drug Library performed with Autodock Vina v4.2 software [20]. Due to the lack of information regarding binding pocket, blinddocking performed as suggested by Vaque et al., 2006 [21]. The virtual screening executed on two separate servers, High-Performance Computing Server (HPC) and ParamShavak Server (PSS). The exhaustiveness 48 and separate grid size set for each 27 receptor molecules in Autodock Vina config file.

\section{Data Analysis}

The Binding energy for each drug ligand exported for analysis from the Autodock Vina v4.2 software and need-based analyses performed manually using python script and excel software. Drug target interaction analysed with Ligplot ${ }^{+}$[22].

\section{Results}

Drug repurposing study for FDA approved 1735 drug molecules $<700$ MW included in the present study. The molecular docking accomplished against a total of 24 modeled proteins and 3 PDB structures followed by seven-stage screening to determine the best combination for COVID19 treatment.

In the first stage, a total of 4,68,650 docking solutions obtained from Molecular docking of approved 1735 drug molecules against 27 COVID19 proteins. From Autodock Vina output, one best pose was extracted from out of 10 poses in the second stage and 46,865 pose data utilized for further analysis (Table 2). In stage three screening step, the top 20 drug molecules based on binding energy were separated for each COVID19 Protein. Which lead to 540 drugs with a potential range of docking scores (Table $1 \& 2$, Figure 1). The redundant drugs removed with the merger of the top 20 dock score for all COVID19 protein together yields 133 unique drugs from the 540 drugs (Supplementary 
material). All the 133 drugs were manually inspected for pharmacological activity reported in Drugbank. Consequently, 18 anticancer drugs, 6 anti-inflammatory, 7 anti-HIV, 8 anti-HCV, 6 drugs for lung disease, 3 drugs for anti-parasitic activity, 2 anti-migraine and few other activities reported. The anti-cancer drugs and drugs with clinically major side effects were omitted. The remaining 35 drugs were selected for further comparative analysis. This included anti-HIV, anti-HCV, antiInflammatory, Lung disease, anti-migraine activity, and anti-parasitic activity. Along with nine clinically reported promising anti-COVD19 drugs also included for further analysis.

Table 2: Process flow for screening

\begin{tabular}{clccc}
\hline Stage & Process & $\begin{array}{c}\text { Targets } \\
\text { included }\end{array}$ & $\begin{array}{c}\text { Drugs } \\
\text { included }\end{array}$ & Remarks \\
\hline 1 & Library creation and Docking & 27 & 1735 & $\begin{array}{c}\text { Obtained total of 468650 } \\
\text { docking solutions }\end{array}$ \\
2 & $\begin{array}{l}\text { Selection of best pose for each } \\
\text { drug }\end{array}$ & 27 & 1735 & 46865 docking solutions \\
3 & $\begin{array}{l}\text { Selection of top 20 drugs for } \\
\text { each target }\end{array}$ & 27 & 1735 & 540 drugs molecules \\
screened
\end{tabular}

The binding energy of selected 35 compounds graded in five grades displaying dark green, light green, yellow, orange and red for easy comparison. Concerning anti-COVID19 activity against single and multiple target analysis, the best results obtained for 8 drugs from anti-HCV followed by 7 antiHIV and 3 currently clinically used drugs. In addition to that, 1 drug from an anti-inflammatory and anti-migraine group also had a good docking score. The overall analysis presented the comparatively better scoring of the selected drug in this study over currently clinically applied drugs for COVID19 treatment (Figure 1) To narrow down the number of drug combinations and removing drug acting on the same target binding energy replaced with docking rank. Based on dock rank 10 drugs selected for further analysis (Figure 2). To reduce the drug for treatment and avoid duplication, the drug acting on the same target was removed. Consequently, the combination of Elbasvir, Ledipasvir, Paritaprevir, Velpatasvir, Antrafenine Ergotamine drug figured out as a potential cocktail for COVID19 treatment (Figure 3). 


\section{Comparative binding interaction of drugs against HCV and COVID19}

To analyse the comparative binding interaction with HCV targets for anti-COVID19 receptors, three targets of HCV NS3 Helicase, NS5B RNA-dependent RNA polymerase and NS3/4A S168A protease docked against 26 potential drugs along with known inhibitors. The result indicated, Chloroquine antiplasmodial drug computationally significant for three HCV receptors but the same is less significant for COVID19 (Figure 4). Similarly, anti-HIV drugs Dolutegravir and Maraviroc had considerable binding against HCV targets but less potential with COVID19 receptors. Conversely, Elbasvir, Ledipasvir reacted with related potential against Helicase, RdRp and Protease from both virus HCV and COVID19. This projected the potential of anti-HCV drugs for inhibition of the COVID19 virus. While ergotamine used in the present study proposes significant binding against all three COVID19 proteins but no promising binding evident with HCV. This suggests the presence of a specific binding site present in COVID19 which might be absent in HCV.

\section{Discussion}

The earlier report of drug repurposing mainly focused on a single target option we represented the holistic approach of targeting all the viral proteins for effective anti-COVID19 activity. The combination suggested will effective on the nonstructural proteins, structural proteins of COVID19 and also included drug the anti-inflammatory with synergic anti-COVID19 activity. Comparative computational analysis of the proposed drug is superior to currently clinically used drug combinations. Which, reflects the possibility of the promising effect of the proposed drug combination in further clinical applications.

Ledipasvir is an inhibitor of the Hepatitis C Virus (HCV) Non-Structural Protein 5A (NS5A)[23]. This protein is crucial for viral RNA replication and assembly of HCV virions. Ledipasvir and Velpatasvir were reported promising in one of the recent studies in virtual screening [24]. Clinical trials against anti-HCV suggest the Ledipasvir treatment significantly improves the patients within one to twelve weeks $[25,26]$.

Similarly, Velpatasvir acts as a defective substrate for NS5A (Non-Structural Protein 5A) sharing a similar function as Ledipasvir. The preclinical study shown the patient having an infection of HCV genotype 1 to 6 can be treated with Velpatasvir [27].

Elbasvir was primarily used for the treatment of HCV. However, some trials are also carried out for using Elbasvir in the treatment of COVID19 [28]. Paritaprevir reported as an inhibitor of COVID19 Protease [29]. No reports are available for anti-COVD19 effectively of Antrafenine and Ergotamine. 
As COVID19 and HCV both are positive-strand RNA viruses and possess a similar mechanism for the creation of viral protein in the host cells. The drugs effective against HCV can have a similar mode of inhibition for the viral protein synthesis mechanism. The lesser effectively of the anti-HIV drugs are due to differential mechanism for synthesis of viral protein. Recently, Chen et al., (2020) reported the clinical study using HCV protease inhibitor Danoprevir to treat naive and experienced COVID-19 patients which are supportive of our hypothesis of using the anti-HCV drugs for antiCOID19 treatment.

\section{Conclusion}

There is an urgent need for anti-COVID-19 drugs to address the global medical emergency. Several drugs are currently employed with empirical clinical knowledge along with some contradictions. Many drug repurposing reports are available but those are mainly concentrated on a single target. The present study directed with a holistic approach of targeting multiple COVID19 proteins with a mixture of FDA approved drugs. The proposed blend drugs include Elbasvir, Ledipasvir, Paritaprevir, and Velpatasvir which currently used for HCV treatment. Inclusion anti-inflammatory Antrafenine and anti-migraine Ergotamine drugs can be effective for dual action of inflammation reduction and COVID19 inhibition. The anticipated combination of drugs acting on both non-structural and structural proteins therefore, can be able to reduce the COVID19 infection process and also reduce viral multiplication. The present study can be immediacy explore further by medical, pharm and research experts effectively to find out the best strategy for anti-COVID19 treatment.

\section{Acknowledgment}

The authors thank Gujarat State Biotechnology Mission (GSBTM) and Gujarat Council on Science and Technology (GUJCOST) and Gujarat Biotechnology Research Centre (GBRC), Department of Science \& Technology (DST), Government of Gujarat, India for computation and technical support.

\section{Data availability}

The docking structures, binding energy results, and poses are available on http://covid19.vnsgusurat.in database. Supplementary file contains all Protein-ligand Interaction poses.

\section{Declaration of competing for interest}

The author declares that there is no conflict of interest in this work. 


\section{Reference}

[1] Updated rapid risk assessment from ECDC on the novel coronavirus disease 2019 (COVID19) pandemic: increased transmission in the EU/EEA and the UK, Eurosurveillance. (2020). https://doi.org/10.2807/1560-7917.es.2020.25.10.2003121.

[2] A.E. Gorbalenya, Severe acute respiratory syndrome-related coronavirus-The species and its viruses, a statement of the Coronavirus Study Group, BioRxiv. (2020).

[3] W.H. Organization, WHO Director-General's opening remarks at the media briefing on COVID-19-11 March 2020, Geneva, Switz. (2020).

[4] D.S. Hui, E. I Azhar, T.A. Madani, F. Ntoumi, R. Kock, O. Dar, G. Ippolito, T.D. Mchugh, Z.A. Memish, C. Drosten, A. Zumla, E. Petersen, The continuing 2019-nCoV epidemic threat of novel coronaviruses to global health - The latest 2019 novel coronavirus outbreak in Wuhan, China, Int. J. Infect. Dis. 91 (2020) 264-266. https://doi.org/10.1016/j.ijid.2020.01.009.

[5] D. of V.D. National Center for Immunization and Respiratory Diseases (NCIRD), C. for D.C. and Prevention, Interim Clinical Guidance for Management of Patients with Confirmed Coronavirus Disease (COVID-19), Centers Dis. Control Prev. (2020). https://doi.org/10.1164/rccm.201908-1581ST.

[6] E. Dong, H. Du, L. Gardner, An interactive web-based dashboard to track COVID-19 in realtime, Lancet Infect. Dis. 0 (2020). https://doi.org/10.1016/S1473-3099(20)30120-1.

[7] Worldometer, Coronavirus Cases, Worldometer. (2020) 1-22. https://doi.org/10.1101/2020.01.23.20018549V2.

[8] N. Chen, M. Zhou, X. Dong, J. Qu, F. Gong, Y. Han, Y. Qiu, J. Wang, Y. Liu, Y. Wei, J. Xia, T. Yu, X. Zhang, L. Zhang, Epidemiological and clinical characteristics of 99 cases of 2019 novel coronavirus pneumonia in Wuhan, China: a descriptive study, Lancet. 395 (2020) 507513. https://doi.org/10.1016/S0140-6736(20)30211-7.

[9] H. Lu, Drug treatment options for the 2019-new coronavirus (2019-nCoV), Biosci. Trends. 13 (2020) 2019-2020. https://doi.org/10.5582/bst.2020.01020.

[10] S. Lin, R. Shen, J. He, X. Li, X. Guo, Molecular modeling evaluation of the binding effect of ritonavir, lopinavir and darunavir to severe acute respiratory syndrome coronavirus 2 proteases, BioRxiv. (2020). https://doi.org/10.1101/2020.01.31.929695.

[11] L. Vinet, A. Zhedanov, Fast Identification of Possible Drug Treatment of Coronavirus Disease -19 (COVID-19) Through Computational Drug Repurposing Study Junmei, Clim. Chang. 2013 - Phys. Sci. Basis. (2010). https://doi.org/10.1088/1751-8113/44/8/085201.

[12] A.A. Elfiky, Anti-HCV, nucleotide inhibitors, repurposing against COVID-19., Life Sci. 248 (2020) 117477. https://www.unboundmedicine.com/medline/citation/32119961/Anti_HCV_nucleotide_inhibit ors_repurposing_against_COVID_19_.

[13] Z. Jin, X. Du, Y. Xu, Y. Deng, M. Liu, Y. Zhao, B. Zhang, X. Li, L. Zhang, Y. Duan, J. Yu, L. Wang, K. Yang, F. Liu, T. You, X. Liu, X. Yang, F. Bai, H. Liu, X. Liu, L.W. Guddat, G. Xiao, C. Qin, Z. Shi, H. Jiang, Z. Rao, H. Yang, Structure-based drug design, virtual screening and high-throughput screening rapidly identify antiviral leads targeting COVID-19, BioRxiv. 
(2020). https://doi.org/10.1101/2020.02.26.964882.

[14] R.N. Kirchdoerfer, A.B. Ward, Structure of the SARS-CoV nsp12 polymerase bound to nsp7 and nsp8 co-factors., Nat. Commun. 10 (2019) 2342. https://doi.org/10.1038/s41467-01910280-3.

[15] D. Wrapp, N. Wang, K.S. Corbett, J.A. Goldsmith, C.-L. Hsieh, O. Abiona, B.S. Graham, J.S. McLellan, Cryo-EM structure of the 2019-nCoV spike in the prefusion conformation, Science (80-. ). 367 (2020) 1260-1263. https://doi.org/10.1126/science.abb2507.

[16] J. Yang, Y. Zhang, I-TASSER server: New development for protein structure and function predictions, Nucleic Acids Res. (2015). https://doi.org/10.1093/nar/gkv342.

[17] N.M. O'Boyle, M. Banck, C.A. James, C. Morley, T. Vandermeersch, G.R. Hutchison, Open Babel: An open chemical toolbox., J. Cheminform. 3 (2011) 33. https://doi.org/10.1186/17582946-3-33.

[18] G. Morris, R. Huey, W. Linkstrom, M. Sanner, R. Belew, D. Goodsell, Olson, AutoDock4 and AutoDockTools4: Automated Docking with Selective Receptor Flexibility, J. Comput. Chem. (2010). https://doi.org/10.1002/jcc.

[19] D.S. Wishart, Y.D. Feunang, A.C. Guo, E.J. Lo, A. Marcu, J.R. Grant, T. Sajed, D. Johnson, C. Li, Z. Sayeeda, N. Assempour, I. Iynkkaran, Y. Liu, A. Maclejewski, N. Gale, A. Wilson, L. Chin, R. Cummings, Di. Le, A. Pon, C. Knox, M. Wilson, DrugBank 5.0: A major update to the DrugBank database for 2018, Nucleic Acids Res. (2018).

https://doi.org/10.1093/nar/gkx1037.

[20] O. Trott, A.J. Olson, AutoDock Vina: improving the speed and accuracy of docking with a new scoring function, efficient optimization, and multithreading., J. Comput. Chem. 31 (2010) 455-461. https://doi.org/10.1002/jcc.21334.

[21] M. Vaque, A. Arola, C. Aliagas, G. Pujadas, BDT: An easy-to-use front-end application for automation of massive docking tasks and complex docking strategies with AutoDock, in: Bioinformatics, 2006: pp. 1803-1804. https://doi.org/10.1093/bioinformatics/btl197.

[22] R.A. Laskowski, M.B. Swindells, LigPlot+: Multiple Ligand-Protein Interaction Diagrams for Drug Discovery, J. Chem. Inf. Model. 51 (2011) 2778-2786. https://doi.org/10.1021/ci200227u.

[23] G.M. Keating, Ledipasvir/Sofosbuvir: A Review of Its Use in Chronic Hepatitis C, Drugs. 75 (2015) 675-685. https://doi.org/10.1007/s40265-015-0381-2.

[24] Y.W. Chen, C.P.B. Yiu, K.Y. Wong, Prediction of the SARS-CoV-2 (2019-nCoV) 3C-like protease (3CLpro) structure: virtual screening reveals velpatasvir, ledipasvir, and other drug repurposing candidates [version 1; peer review: 3 approved], F1000Research. 9 (2020). https://doi.org/10.12688/f1000research.22457.1.

[25] Z.M. Younossi, M. Stepanova, P. Marcellin, N. Afdhal, K. V Kowdley, S. Zeuzem, S.L. Hunt, Treatment with ledipasvir and sofosbuvir improves patient-reported outcomes: Results from the ION-1, -2, and -3 clinical trials., Hepatology. 61 (2015) 1798-1808. https://doi.org/10.1002/hep.27724.

[26] Y. Waheed, Ledipasvir and sofosbuvir: Interferon free therapy for HCV genotype 1 infection, World J. Virol. 4 (2015) 33. https://doi.org/10.5501/wjv.v4.i1.33. 
[27] E. Mogalian, P. German, B.P. Kearney, C.Y. Yang, D. Brainard, J. Link, J. McNally, L. Han, J. Ling, A. Mathias, Preclinical Pharmacokinetics and First-in-Human Pharmacokinetics, Safety, and Tolerability of Velpatasvir, a Pangenotypic Hepatitis C Virus NS5A Inhibitor, in Healthy Subjects, Antimicrob. Agents Chemother. 61 (2017).

https://doi.org/10.1128/AAC.02084-16.

[28] T. Asselah, H. Reesink, J. Gerstoft, V. de Ledinghen, P.J. Pockros, M. Robertson, P. Hwang, E. Asante-Appiah, J. Wahl, B.-Y. Nguyen, E. Barr, R. Talwani, L. Serfaty, Efficacy of elbasvir and grazoprevir in participants with hepatitis $C$ virus genotype 4 infection: A pooled analysis., Liver Int. 38 (2018) 1583-1591. https://doi.org/10.1111/liv.13727.

[29] M.A. Alamri, M. Tahir ul Qamar, S.M. Alqahtani, Pharmacoinformatics and Molecular Dynamic Simulation Studies Reveal Potential Inhibitors of SARS-CoV-2 Main Protease 3CL ${ }^{\text {pro }}$, (2020) 1-16. https://doi.org/10.20944/preprints202002.0308.v1. 


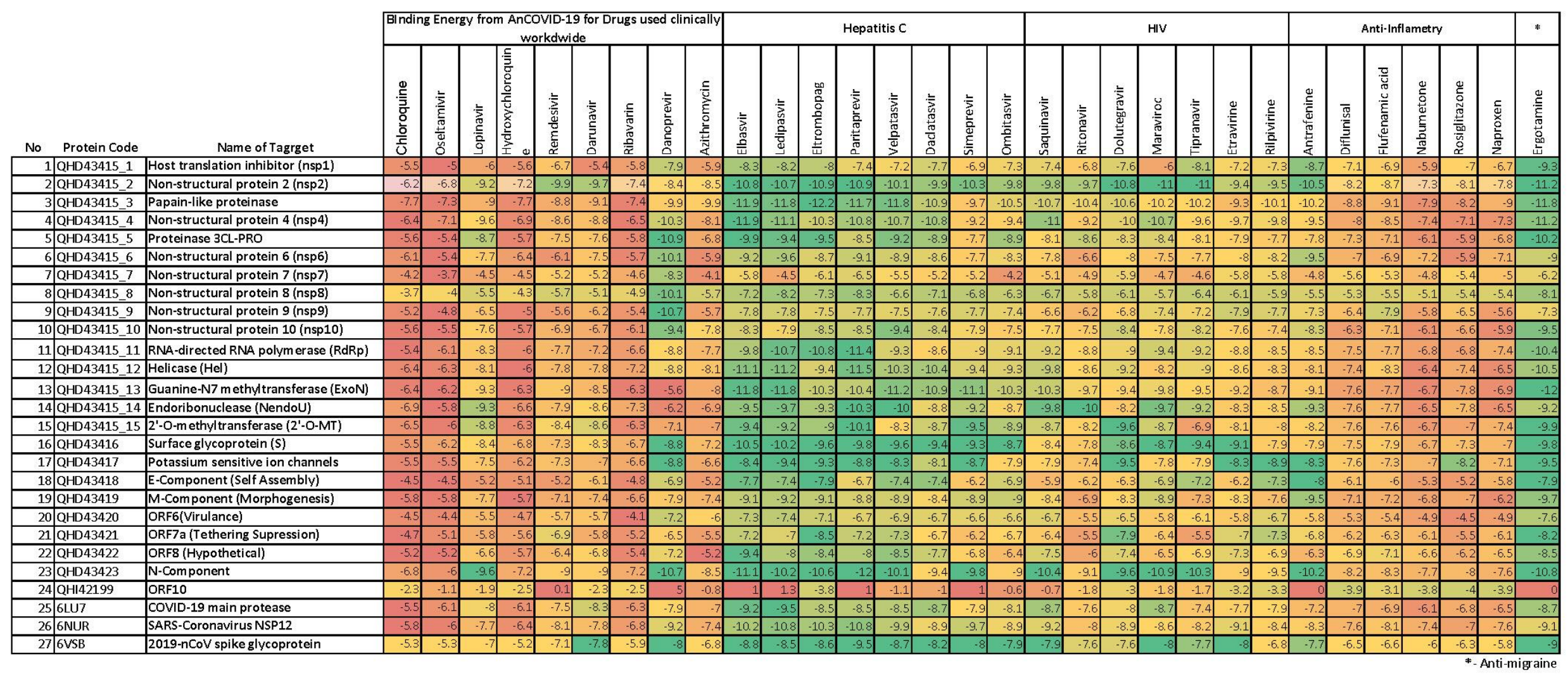

The Green colored cell contains the lowest binding energy for each row followed by the greenish-yellow, yellow, orange and red color. The lowest binding energy represents the firm binding of ligand and protein molecules. The Comparative analysis establishes the drugs used for the treatment of Hepatitis $\mathrm{C}$ represented a more number of greenish cells. This indicates these drugs stay on top throughout the library used in the present study. The Elbasvir, Ledipasvir, Paritaprevir, Velpatasvir from Anti-HCV followed by Anti-HIV drugs Saquinavir, Ritonavir, Dolutegravir, Maraviroc, Tipranavir, Etravirine and Rilpivirine having good binding energy one or more targets. Only one drug, Antrafenine used for anti-inflammatory and Ergotamine used for anti-migraine activity shown significant activity against the different targets. However, the drugs used worldwide for clinical purposes except for Danoprevir and Lopinavir have shown poor binding with receptor molecules used in the study.

Figure 1: Comparative analysis of 35 drug molecules against 27 COVID19 proteins based on binding energy 


\begin{tabular}{|c|c|c|c|c|c|c|c|c|c|c|c|c|}
\hline \multirow[b]{2}{*}{ No } & \multirow[b]{2}{*}{ Protein Code } & \multirow[b]{2}{*}{ Name of Tagrget } & \multicolumn{5}{|c|}{ Anti-HCV } & \multicolumn{3}{|c|}{ Anti-HIV } & \multirow{2}{*}{ 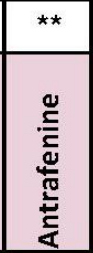 } & \multirow{2}{*}{ 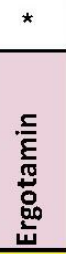 } \\
\hline & & & 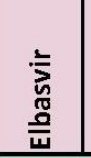 & 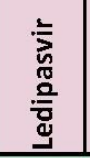 & 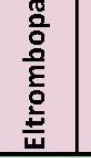 & 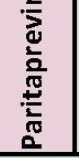 & 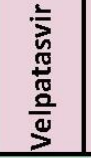 & 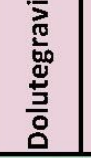 & 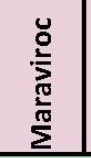 & 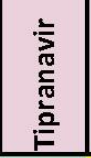 & & \\
\hline 1 & QHD43415_1 & Host translation inhibitor (nsp1) & 11 & 13 & 22 & 97 & 157 & 62 & 884 & 16 & 4 & 1 \\
\hline 2 & QHD43415_2 & Non-structural protein 2 (nsp2) & 22 & 25 & 13 & 15 & 64 & 20 & 8 & 6 & 37 & 4 \\
\hline 3 & QHD43415_3 & Papain-like proteinase & 6 & 8 & 3 & 12 & 9 & 61 & 120 & 114 & 117 & 7 \\
\hline 4 & QHD43415_4 & Non-structural protein 4 (nsp4) & 4 & 17 & 55 & 31 & 34 & 86 & 33 & 139 & 164 & 14 \\
\hline 5 & QHD43415_5 & Proteinase 3 CL-PRO & 2 & 7 & 5 & 55 & 15 & 81 & 64 & 114 & 193 & 1 \\
\hline 6 & QHD43415_6 & Non-structural protein 6 (nsp6) & 9 & 3 & 31 & 11 & 18 & 121 & 281 & 186 & 5 & 12 \\
\hline 7 & QHD43415_7 & Non-structural protein 7 (nsp7) & 103 & 1078 & 43 & 13 & 232 & 74 & 855 & 924 & 748 & 26 \\
\hline 8 & QHD43415_8 & Non-structural protein 8 (nsp8) & 14 & 2 & 7 & 1 & 59 & 147 & 319 & 78 & 462 & 3 \\
\hline 9 & QHD43415_9 & Non-structural protein 9 (nsp9) & 12 & 10 & 33 & 19 & 38 & 177 & 44 & 66 & 54 & 51 \\
\hline 10 & QHD43415_10 & Non-structural protein 10 (nsp10) & 62 & 128 & 35 & 37 & 5 & 48 & 148 & 68 & 58 & 3 \\
\hline 11 & QHD43415_11 & RNA-directed RNA polymerase (RdRp) & 22 & 4 & 3 & 2 & 56 & 97 & 46 & 61 & 198 & 7 \\
\hline 12 & \begin{tabular}{|l|l|} 
QHD43415_12 \\
\end{tabular} & Helicase (Hel) & 4 & 3 & 37 & 2 & 12 & 63 & 270 & 78 & 312 & 8 \\
\hline 13 & QHD43415_13 & Guanine-N7 methyltransferase (ExoN) & 3 & 2 & 42 & 37 & 10 & 166 & 88 & 137 & 236 & 1 \\
\hline 14 & QHD43415_14 & Endoribonuclease (NendoU) & 34 & 26 & 47 & 6 & 12 & 267 & 25 & 57 & 44 & 56 \\
\hline 15 & QHD43415_15 & 2'-0-methyltransferase (2'-O-MT) & 35 & 6 & 76 & 7 & 289 & 18 & 145 & 955 & 311 & 12 \\
\hline 16 & QHD43416 & Surface glycoprotein (S) & 2 & 4 & 28 & 20 & 30 & 182 & 150 & 37 & 449 & 16 \\
\hline 17 & QHD43417 & Potassium sensitive ion channels & 66 & 5 & 6 & 31 & 95 & 3 & 228 & 172 & 81 & 2 \\
\hline 18 & QHD43418 & E-Component (Self Assembly) & 11 & 17 & 7 & 88 & 21 & 207 & 55 & 29 & 3 & 6 \\
\hline 19 & QHD43419 & M-Component (Morphogenesis) & 13 & 5 & 11 & 33 & 23 & 81 & 19 & 369 & 3 & 2 \\
\hline 20 & QHD43420 & ORF6(Virulance) & 9 & 7 & 14 & 57 & 28 & 91 & 286 & 164 & 281 & 3 \\
\hline 21 & QHD43421 & ORF7a (Tethering Supression) & 65 & 98 & 1 & 62 & 48 & 10 & 321 & 1008 & 141 & 3 \\
\hline 22 & QHD43422 & ORF8 (Hypothetical) & 1 & 11 & 5 & 13 & 4 & 62 & 381 & 152 & 497 & 3 \\
\hline 23 & QHD43423 & N-Component & 10 & 45 & 20 & 2 & 54 & 139 & 15 & 36 & 44 & 17 \\
\hline 24 & QHI42199 & ORF10 & 1583 & 1584 & 19 & 6 & 1528 & 422 & 1406 & 1425 & 1562 & 5 \\
\hline 25 & 6LU7 & COVID-19 main protease & 14 & 3 & 57 & 63 & 64 & 162 & 36 & 396 & 496 & 34 \\
\hline 26 & 6NUR & SARS-Coronavirus NSP12 & 9 & 1 & 6 & 2 & 16 & 78 & 140 & 265 & 228 & 10 \\
\hline 27 & 6VSB & 2019-nCoV spike glycoprotein & 5 & 10 & 8 & 1 & 7 & 75 & 29 & 52 & 55 & 2 \\
\hline & & Total Drug Score & 2131 & 3122 & 634 & 723 & 2928 & 3000 & 6396 & 7104 & 6783 & 309 \\
\hline
\end{tabular}

All the 35 drug molecules screened based on their docking score/rank and selected molecules that lie in the range of 1 to 10. These molecules were marked with having a yellow background in cells. Total 10 drugs found having a lesser docking score. This includes 5 drugs of Anti-HCV followed by 3 Anti-HIV and 1 for anti-inflammatory and anti-migraine.

Figure 2: Selected drugs having significant docking score $(\leq 10)$ 


\begin{tabular}{|c|c|c|c|c|c|c|c|c|}
\hline \multirow[b]{2}{*}{ No } & \multirow[b]{2}{*}{ Protein Code } & \multirow[b]{2}{*}{ Name of Tagrget } & \multicolumn{4}{|c|}{ Anti-HCV } & \multirow{2}{*}{ 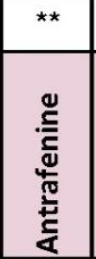 } & \multirow{2}{*}{ 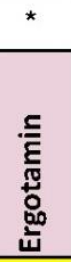 } \\
\hline & & & 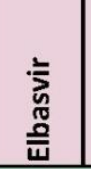 & 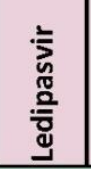 & 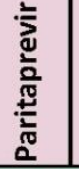 & 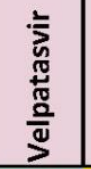 & & \\
\hline 1 & QHD43415_1 & Host translation inhibitor (nsp1) & 11 & 13 & 97 & 157 & 4 & 1 \\
\hline 2 & QHD43415_2 & Non-structural protein 2 (nsp2) & 22 & 25 & 15 & 64 & 37 & 4 \\
\hline 3 & QHD43415_3 & Papain-like proteinase & 6 & 8 & 12 & 9 & 117 & 7 \\
\hline 4 & QHD43415_4 & Non-structural protein 4 (nsp4) & 4 & 17 & 31 & 34 & 164 & 14 \\
\hline 5 & QHD43415_5 & Proteinase 3CL-PRO & 2 & 7 & 55 & 15 & 193 & 1 \\
\hline 6 & QHD43415_6 & Non-structural protein 6 (nsp6) & 9 & 3 & 11 & 18 & 5 & 12 \\
\hline 7 & QHD43415_7 & Non-structural protein 7 (nsp7) & 103 & 1078 & 13 & 232 & 748 & 26 \\
\hline 8 & QHD43415_8 & Non-structural protein 8 (nsp8) & 14 & 2 & 1 & 59 & 462 & 3 \\
\hline 9 & QHD43415_9 & Non-structural protein 9 (nsp9) & 12 & 10 & 19 & 38 & 54 & 51 \\
\hline 10 & QHD43415_10 & Non-structural protein 10 (nsp10) & 62 & 128 & 37 & 5 & 58 & 3 \\
\hline 11 & QHD43415_11 & RNA-directed RNA polymerase (RdRp) & 22 & 4 & 2 & 56 & 198 & 7 \\
\hline 12 & QHD43415_12 & Helicase (Hel) & 4 & 3 & 2 & 12 & 312 & 8 \\
\hline 13 & QHD43415_13 & Guanine-N7 methyltransferase (ExoN) & 3 & 2 & 37 & 10 & 236 & 1 \\
\hline 14 & QHD43415_14 & Endoribonuclease (NendoU) & 34 & 26 & 6 & 12 & 44 & 56 \\
\hline 15 & QHD43415_15 & 2'-O-methyltransferase (2'-O-MT) & 35 & 6 & 7 & 289 & 311 & 12 \\
\hline 16 & QHD43416 & Surface glycoprotein (S) & 2 & 4 & 20 & 30 & 449 & 16 \\
\hline 17 & QHD43417 & Potassium sensitive ion channels & 66 & 5 & 31 & 95 & 81 & 2 \\
\hline 18 & QHD43418 & E-Component (Self Assembly) & 11 & 17 & 88 & 21 & 3 & 6 \\
\hline 19 & QHD43419 & M-Component (Morphogenesis) & 13 & 5 & 33 & 23 & 3 & 2 \\
\hline 20 & QHD43420 & ORF6(Virulance) & 9 & 7 & 57 & 28 & 281 & 3 \\
\hline 21 & QHD43421 & ORF7a (Tethering Supression) & 65 & 98 & 62 & 48 & 141 & 3 \\
\hline 22 & QHD43422 & ORF8 (Hypothetical) & 1 & 11 & 13 & 4 & 497 & 3 \\
\hline 23 & QHD43423 & N-Component & 10 & 45 & 2 & 54 & 44 & 17 \\
\hline 24 & QHI42199 & ORF10 & 1583 & 1584 & 6 & 1528 & 1562 & 5 \\
\hline 25 & 6LU7 & COVID-19 main protease & 14 & 3 & 63 & 64 & 496 & 34 \\
\hline 26 & 6NUR & SARS-Coronavirus NSP12 & 9 & 1 & 2 & 16 & 228 & 10 \\
\hline 27 & 6VSB & 2019-nCoV spike glycoprotein & 5 & 10 & 1 & 7 & 55 & 2 \\
\hline
\end{tabular}

The drugs from the list removed having overlapping target applicability. The removal identified 6 unique drugs acting on the multiple targets. The combination of these drugs overcomes the lower affinity of the drug molecules to the specific receptors targeting all the proteins available in the present study. Ergotamine shown good binding against 18 receptors, followed by Lepidasvir, Elbasivir and Pariptapevir respectively against 16, 12 and 9 receptors. While velpatasvir and anterafenine showed binding against 5 which might be very useful in a multi-drug combinatorial approach.

Figure 3: Suggested best combination for multi-target approach against COVID-19 


\begin{tabular}{|c|c|c|c|c|c|c|c|c|c|c|c|c|c|c|c|c|c|c|c|c|c|c|c|c|c|c|c|c|c|c|}
\hline \multirow[b]{2}{*}{ No } & \multirow[b]{2}{*}{$\begin{array}{l}\text { Name of } \\
\text { Organism }\end{array}$} & \multirow[b]{2}{*}{ Name of Tagrget } & \multirow[b]{2}{*}{ Protein Code } & \multicolumn{10}{|c|}{ Binding Energy from Ancovid-19 for Drugs used clinically } & \multicolumn{8}{|c|}{ Hepatitis C } & \multicolumn{7}{|c|}{ HIV } & \multirow[b]{2}{*}{ 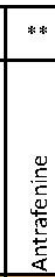 } & \multirow[b]{2}{*}{ 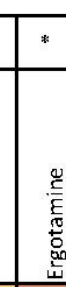 } \\
\hline & & & & 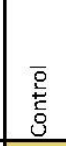 & 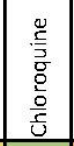 & 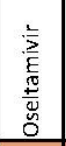 & 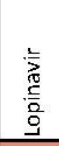 & 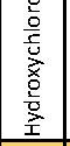 & 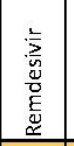 & 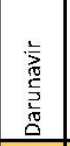 & 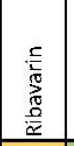 & 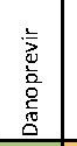 & 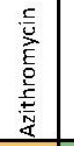 & 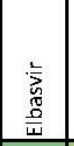 & 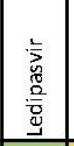 & 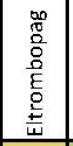 & 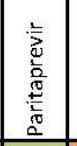 & 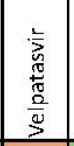 & 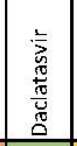 & 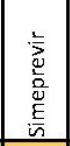 & 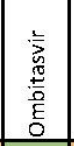 & 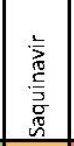 & 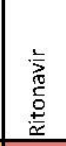 & 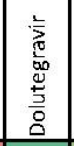 & 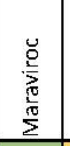 & 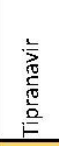 & 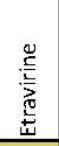 & 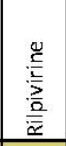 & & \\
\hline 1 & $\mathrm{HCV}$ & NS3 Helicase & $4 W \times R$ & -8.9 & -9.9 & -6.7 & -6.2 & -8.3 & -8.2 & -8.2 & -8.4 & -9.9 & -7.9. & -10.6 & -10 & -8.9 & -9.6 & -6.9 & -10 & -8.2 & -10.1 & -7.7 & -5.8 & -11.3 & -10.1 & -8.5 & -9.1 & -9.1 & -8.5 & -6.4 \\
\hline 2 & COVID19 & Helicase (Hel) & ahd43415_11 & & $|-6.4|$ & -6.3 & -8.1 & -6 & $\mid-7.8$ & -7.8 & $\mid-7.2$ & -8.8 & -8.1 . & $\mid-11.1$ & $\mid-11.2$ & $\mid-9.4$ & -11.5 & -10.3 & $|-10.4|$ & -9.4 & $\mid-9.3$ & $|-9.8|$ & $\mid-8.6$ & $\mid-9.2$ & -8.2 & -9 & -8.6 & $\mid-8.3$ & $\mid-8.1$ & $|-10|$ \\
\hline 3 & $\mathrm{HCV}$ & $\begin{array}{l}\text { NS5B RNA-dependent RNA } \\
\text { polymerase }\end{array}$ & 5QJ0 & -10 & -11.1 & -6.2 & -6.8 & -9.2 & -8.6 & -10.3 & -8.8 & -10.3 & -8 & -10.5 & -10.2 & -10.7 & -9.4 & -7.5 & -9.1 & -9.5 & -11 & -7.6 & -5.8 & -11.6 & -9.8 & -10.1 & -9.8 & -9.5 & -9 & -6.4 \\
\hline 4 & CovID19 & $\begin{array}{l}\text { RNA-directed RNA } \\
\text { polymerase (RdRp) } \\
\end{array}$ & qhd43415_12 & & -5.4 & -6.1 & -8.3 & -6 & -7.7 & -7.2 & -6.6 & $\mid-8.8$ & -7.7 & $\mid-9.8$ & $|-10.7|$ & -10.8 & -11.4 & $\mid-9.3$ & $\mid-8.6$ & -9 & $|-9.1|$ & $\mid-9.2$ & $\mid-8.8$ & -9 & $\mid-9.4$ & -9.2 & -8.8 & \begin{tabular}{|l|}
-8.5 \\
\end{tabular} & -8.5 & $\mid-11$ \\
\hline 5 & $\mathrm{HCV}$ & NS3/4A D168A protease & 6PIV & -11.7 & -9.1 & -5.7 & -6 & -7.9 & -7.7 & -7 & -6.5 & \begin{tabular}{|l|}
-7.9 \\
\end{tabular} & -6.5 & -9 & \begin{tabular}{|l|}
-8.7 \\
\end{tabular} & \begin{tabular}{|l|}
-7.6 \\
\end{tabular} & -7.6 & \begin{tabular}{|l|}
-7.8 \\
\end{tabular} & -8 & -7.9 & -9.4 & \begin{tabular}{|l|}
-7.2 \\
\end{tabular} & -5.1 & \begin{tabular}{|l|}
-9.7 \\
\end{tabular} & -8.7 & -7.6 & -7.8 & \begin{tabular}{|l|}
-7.9 \\
\end{tabular} & -6.6 & -6.2 \\
\hline 6 & COVID19 & Proteinase 3CL-PRO & qhd43415_5 & & -5.6 & -5.4 & -8.7 & \begin{tabular}{|l|}
$\mid-5.7$ \\
\end{tabular} & $\mid-7.5$ & $\mid-7.6$ & -5.8 & $|-10.9|$ & -6.8 & $\mid-9.9$ & $\mid-9.4$ & $\mid-9.5$ & -8.5 & $\mid-9.2$ & $\mid-8.9$ & $|-7.7|$ & $|-8.9|$ & $-8.1 \mid$ & $\mid-8.6$ & -8.3 & -8.4 & -8.1 & -7.9 & \begin{tabular}{|l|}
$\mid-7.7$ \\
\end{tabular} & -7.8 & -10 \\
\hline
\end{tabular}

The results graded on five color green for lowest binding energy followed by greenish-yellow, yellow, orange and red for the highest binding energy for each receptor.

Figure 4: Comparison of HCV targets as a reference against COVID19. 